\title{
The Effect of Fluorescent Strigolactone Mimics on the Development of Phytophathogenic Fungi ${ }^{+}$
}

\author{
Ioana-Alexandra Bala ${ }^{1}$, Florentina Georgescu ${ }^{2}$, Emilian Georgescu ${ }^{2}$, \\ Diana Constantinescu-Aruxandei $1, *$ and Florin Oancea ${ }^{1, *}$ \\ 1 National Institute for Research \& Development in Chemistry and Petrochemistry - ICECHIM, \\ 060021 Bucharest, Romania; ioana-alexandra.bala@icechim.ro \\ 2 Teso Spec Srl, Str. Muncii 53, 915200 Fundulea, Calarasi, Romania; florentina_fg@yahoo.com (F.G.); \\ g_emilian@yahoo.com (E.G.) \\ * Correspondence: diana.constantinescu@icechim.ro (D.C.-A.); florin.oancea@icechim.ro (F.O.) \\ + Presented at the 16th International Symposium "Priorities of Chemistry for a Sustainable Development" \\ PRIOCHEM, Bucharest, Romania, 28-30 October 2020.
}

Published: 9 November 2020

Keywords: strigolactone; phytophatogenic fungi; radial growth; hyphal branching

Strigolactones (SLs) are organic molecules synthesized by plants that have two main functions, acting as plant hormones and regulating numerous aspects regarding plant development and various stress-related functions, as well as signaling molecules in the rhizosphere [1]. Due to their biological activities, they could be used as plant biostimulants, but the natural SLs have a complex structure that is difficult to synthesize. Therefore, SL analogoues and later SL mimics that preserve the activity of natural SLs have been synthesized [2]. In this study, we tested the bioactivity of new SL fluorescent mimics on two strains of phytophatogenic fungi. The fungal strains were Fusarium graminearum and Rhizoctonia solani, which were grown on Potato Dextrose Agar (PDA) medium at 28 ${ }^{\circ} \mathrm{C}$, for 5 days. A piece of agar was taken and placed in the center of Petri dishes of either water agar or Poloxamer 407 medium containing different solutions of fluorescent SL mimics (SL-20 and SL-21) incorporated in different culture media which include agar and Poloxamer 407. To examine the number of hyphal branches and their morphological characteristics in poloxamer and agar cultures, we used a stereomicroscope (OPTIKA, Italy). The diameter of the fungal colonies was measured after 3 days. The radial growth of the two tested fungi was significantly inhibited by the classical SL analogoue GR24, and by the new SL mimics SL-20 and SL-21 at a concentration of 10-5 both in agar and poloxamer. The new SL mimics, especially SL-21, were as efficient as GR24. The highest effect was obtained against $R$. solani. At lower concentrations, the effect was much less pronounced. No significant effect was observed on hyphal branching for neither SL concentration, but GR24 and SL-21, and to a lesser extent SL-20, increased the pigmentation of F. graminearum in the medium with poloxamer. We propose new SL fluorescent mimics that have the same effect as GR24, inducing a stress response and inhibiting phytopathogen growth. The synthesised SL mimics can be used for further in depth investigation of SL-plants and SL-microorganisms interactions based on fluorescence analysis.

Acknowledgments: This work was funded by Project RO-NO 540 STIM 4+/2020, financed by EEA and Norway Grants. 


\section{References}

1. Lopez-Raez, J.A. Strigolactones: Crucial Cues in the Rhizosphere. Mol. Microb. Ecol. Rhizosphere 2013, 381-389, doi:10.1002/9781118297674.ch35.

2. Oancea, F.; Georgescu, E.; Matusova, R.; Georgescu, F.; Nicolescu, A.; Raut, I.; Jecu, M.L.; Vladulescu, M.C.; Deleanu, C. New strigolactone mimics as exogenous signals for rhizosphere organisms. Molecules 2017, 22, 961.

Publisher's note: MDPI stays neutral with regard to jurisdictional claims in published maps and institutional affiliations.

(c) 2020 by the authors. Licensee MDPI, Basel, Switzerland. This article is an open access article distributed under the terms and conditions of the Creative Commons Attribution (CC BY) license (http://creativecommons.org/licenses/by/4.0/). 